\section{rev Psi}

Revista de Psicología (UNLP)

https://revistas.unlp.edu.ar/revpsi

\title{
Validación de un cuestionario de riesgos psicosociales en el trabajo en población trabajadora argentina (COPSOQII-ISTAS21)
}

\author{
María Constanza Zelaschi ${ }^{1,2}$ \\ Luciana Reif ${ }^{2}$ \\ Correspondencia \\ zelaschiunlp@gmail.com \\ Filiaciones institucionales \\ ${ }^{1}$ Facultad de Psicología, Universidad Nacional de \\ La Plata (UNLP, Argentina)
}

\author{
Cecilia Cornelio ${ }^{2}$ \\ Marcelo Amable ${ }^{2}$
}

${ }^{2}$ GESAL - Universidad Nacional de Avellaneda (Argentina)

\section{Resumen}

En este artículo presentaremos la validación del Cuestionario Psicosocial de Copenhague (COPSOQ). La adaptación cultural se realizó sobre la versión larga del COPSOQII -ISTAS21. La validación se implementó sobre una muestra aleatoria de 200 casos, representativa del conglomerado de Ciudad de Buenos Aires, Avellaneda, Morón y Vicente López. Se procedió a realizar análisis de consistencia interna con Alpha de Cronbach y validación de constructo a través de análisis factorial. Los resultados fueron satisfactorios en cuanto a la consistencia interna, aunque con algunos valores bajos para ciertas dimensiones (valores de $\alpha$ entre 0,582 y 0,895); la estructura factorial fue la esperada según el cuestionario original. La validez de criterio evaluada en las correlaciones con las mediciones de salud a partir del cuestionario SF36, fueron satisfactorias El comportamiento del instrumento en población de trabajadores en Argentina ha sido similar a las versiones internacionales.

\section{Palabras clave}

salud laboral | fuerza de trabajo | factores de riesgos psicosociales | validación de cuestionario

Cómo citar

Zelaschi, C. M., Cornelio, C., Reif, L., Amable, M. (2021). Validación de un cuestionario de riesgos psicosociales en el trabajo en población trabajadora argentina (COPSOQII-ISTAS21). Revista de Psicología, 20(2), 2-17. HTTPs://DX.DOI. ORG/10.24215/2422572XE084

\section{Proceso editorial}

Recibido

14 sep. 2020

Aceptado

17 feb. 2021
1 ra decisión

30 nov. 2020

Publicado 20 feb. 2021
ISSN

2422-572X

Licencia

Licencia de Cultura Libre CC-BY 4.0

(Compartir - Adaptar - Atribuir)

Entidad editora

RevPsi es una publicación de la

Facultad de Psicología (Universidad

Nacional de La Plata, Argentina) 


\section{Validação de questionário de riscos psicossociais no trabalho na população trabalhadora da Argentina (COPSOQII-ISTAS21)}

\section{Resumo}

Neste artigo apresentaremos a validação do Questionário Psicossocial de Copenhague (COPSOQ). A adaptação cultural foi realizada na versão longa do COPSOQII -ISTAS21. A validação foi realizada em uma amostra aleatória de 200 casos, representativos do conglomerado da Cidade de Buenos Aires, Avellaneda, Morón e Vicente López. A análise da consistência interna foi realizada com o Alpha de Cronbach e a validação do construto por meio da análise fatorial. Os resultados foram satisfatórios em termos de consistência interna, embora com alguns valores baixos para algumas dimensões (valores de a entre 0,582 e 0,895); a estrutura fatorial foi a esperada de acordo com o questionário original. A validade de critério avaliada nas correlações com as medidas de saúde do questionário SF 36 foi satisfatória. O comportamento do instrumento na população de trabalhadores da Argentina tem sido semelhante às versões internacionais.

\section{Palavras-chave}

saúde ocupacional | força de trabalho | fatores de risco psicossocial | validação do questionário

\section{Validation of a questionnaire for psychosocial risks assessment in working popultation in Argentina (COPSOQII-ISTAS21)}

\section{Abstract}

In this article we will present the validation of the Copenhagen Psychosocial Questionnaire (COPSOQ). The cultural adaptation was carried out on the long version of the COPSOQII -ISTAS21. The validation was carried out on a random sample of 200 cases, representative of the conglomerate of the City of Buenos Aires, Avellaneda, Morón and Vicente López. Internal consistency analysis was performed with Cronbach's Alpha and construct validation through factor analysis. The results were satisfactory in terms of internal consistency, although with some low values for certain dimensions ( $\alpha$ values between 0.582 and 0.895 ); the factorial structure was as expected according to the original questionnaire. The criterion validity evaluated in the correlations with the health measurements from the SF36 questionnaire were satisfactory. The behavior of the instrument in the population of workers in Argentina has been similar to the international versions.

\section{Keywords}

occupational health | work force | occupational psychosocial risks | questionnaire validation 


\section{Aspectos destacados del trabajo}

- El COPSOQ mide la exposición a factores de riesgos psicosociales en el trabajo.

- Es un cuestionario de uso internacional. Permite realizar análisis comparativos entre países.

- Los resultados de la validación del instrumento fueron los esperados.

- El COPSOQ-ARG es un instrumento que permite realizar prevención en salud laboral.

El trabajo es uno de los determinantes de la salud poblacional que mayor impacto tiene sobre las desigualdades sociales en salud (CSDH, 2008). Desde hace décadas que se han introducido en el mundo del trabajo globalizado (Benach et al., 2002; Ferrie, 2001) reformas que flexibilizaron las relaciones laborales y nuevas técnicas de gestión de la fuerza de trabajo (Paoli, 1992). Estas reformas poseen un impacto directo sobre la salud de los trabajadores/as (Virtanen et al., 2005; Amable, 2006; Zelaschi, 2010), lo que genera un creciente nivel de exigencias y compromisos laborales que sitúan a los factores psicosociales del trabajo (Kalimo et al., 1988) como una prioridad para la salud laboral (Benavides et al., 2007). Los factores de riesgo tradicionales surgidos de exposiciones industriales, pierden proporcionalidad ante nuevas dimensiones psicosociales del trabajo. Los riesgos psicosociales han pasado a configurar un nuevo perfil epidemiológico en la exposición de los diversos grupos de trabajadores debido al aumento de la inestabilidad laboral y las exigencias psicosociales derivadas de las nuevas formas de trabajo. Además, el desarrollo creciente del sector servicios con su organización de trabajo más flexible, conlleva un desplazamiento de la subjetividad de los trabajadores/as hacia el centro del desempeño laboral como requerimiento.

Además, en las últimas décadas, la investigación epidemiológica ha podido demostrar empíricamente la relación de los factores psicosociales del trabajo con la salud de los/as trabajadores/as. La exposición a estos factores de riesgo psicosociales incide sobre diversos aspectos de la salud: trastornos y enfermedades psicosomáticas (Landsbergis y Theorell, 2000), problemas osteomusculares (Cardoso et al., 2011), cardiovasculares (Kivimäki et al., 2002; Belkic et al., 2004; Gómez-Ortiz y Moreno, 2009) y/o de ausentismo (Benzoni, 2018; Gimeno et al., 2004). Estos resultados no sólo pusieron de manifiesto la relevancia para la salud pública de estas exposiciones laborales, sino que también rompieron con los límites académicos tradicionales con los que se venía abordando este problema en el ámbito laboral. Las perspectivas predominantes surgidas en los años '60, lograron situar la salud mental en el mundo del trabajo, pero lo hicieron desde los estudios del estrés. En consecuencia, se sucedieron un conjunto de estudios centrados en las características personales de los trabajadores (estudios sobre tipos de personalidad), en el contenido cognitivo de la 
actividad desarrollada (ocupaciones de responsabilidad o categorías de profesionales), aplicando un individualismo metodológico que restringía las intervenciones colectivas de la prevención. Sin embargo, los modelos de factores psicosociales en el trabajo han podido demostrar que la esa exposición es transversal a las ocupaciones, independientemente del contenido de las tareas, porque es consecuencia directa de los rasgos que impone la organización del trabajo en la búsqueda de productividad. Se ha demostrado que la exposición psicosocial no afecta sólo a la salud mental, sino que se manifiesta de forma integral a través de diversos fenómenos del proceso salud-enfermedad. Por lo tanto, desde hace décadas que la evaluación de los factores psicosociales del trabajo es una necesidad y un requerimiento legal en muchos países, en la prevención de riesgos para la salud de los/as trabajadores/as.

En Argentina, la proporción de trabajadores/as que manifiestan estar expuestos a factores de riesgos psicosociales es alarmante: la prevalencia de exposición oscila entre un 40 y un 60\%, según el tipo de riesgo que se trate (Superintendencia de Riesgos del Trabajo, 2009). Sin embargo, todavía existe un retraso en el marco legal preventivo, siendo difícil la equiparación de los riesgos psicosociales con el resto de los riesgos laborales, en cuanto a las responsabilidades patronales de seguridad e higiene. En ese sentido, es necesario que se modernicen los servicios de prevención a través del desarrollo de especialidades de posgrado en distintas disciplinas, que obtengan el reconocimiento legal pertinente para alcanzar una composición interdisciplinaria e integral de dichos servicios. La evaluación de riesgos para implementar programas preventivos en las empresas requiere del desarrollo de métodos válidos y socialmente aceptados. La vigilancia ambiental y epidemiológica de factores psicosociales, no es una excepción. Los procedimientos objetivables, son una herramienta central para la gestión político-técnica que implica la prevención de riesgos del trabajo, ya que facilita la comprensión y el diálogo social en cualquier tipo de organización. Hasta el momento, no existía en el país una metodología probada y validada para identificar, localizar y medir riesgos psicosociales en el ambiente de trabajo. Consideramos que disponer de un método como el que aquí presentamos, de uso internacional desde hace más de 30 años y validado en diversos contextos, será de gran utilidad para mejorar la salud en los ámbitos laborales de nuestro país.

Existen en Latinoamérica diversos esfuerzos para medir las exposiciones laborales, comparativamente entre países (Benavides et al., 2018). Una de las metodologías para la evaluación de riesgos psicosociales más difundidas es el Cuestionario Psicosocial de Copenhage (COPSOQ) (Kristensen et al., 2005) que incorpora los modelos de estrés psicosocial en el trabajo que más evidencias epidemiológicas han aportado de su impacto a la salud (Karasek, 1979; Siegrist et al., 1990). El COPSOQ es un instrumento validado y utilizado en varios países e idiomas y con amplias referencias en estudios científicos (Nolle y Kranich, 2020). También es citado como un método de referencia en documentos de instituciones internacionales, como por ejemplo la Organización Mundial de la Salud o la Agencia Europea para la Seguridad y la Salud en el Trabajo. Se gestiona de forma colaborativa por la COPSOQ International Network, bajo los principios de investigación orientada a la acción, lo que garantiza 
su actualización a los cambios en el mundo del trabajo y al avance del conocimiento científico (Kristensen, 2010).

La versión en castellano (COPSOQ-ISTAS21) ha sido desarrollada y validada por el Instituto Sindical de Trabajo, Ambiente y Salud (ISTAS) de Barcelona, en España (Moncada et. al., 2005; Moncada et al., 2014; Moncada et.al., 2008). Se trata de una metodología para la evaluación y gestión de riesgos psicosociales, que tiene como propósito identificar aquellas características de la organización del trabajo que pueden representar riesgos para la salud y el bienestar de los trabajadores/as, facilitando las propuestas preventivas en un marco participativo (Zárate Castillo y Gómez Gómez, 2012).

La mencionada versión en castellano se ha tomado como base para la adaptación cultural y validación en diferentes países de América Latina (Alvarado et al., 2012; Lima et al., 2019; Ramada-Rodilla et al., 2013). En Argentina, a partir del año 2013 se conformó el grupo COPSOQ-ARG entre la Universidad Nacional de Avellaneda (UNDAV) y la Superintendencia de Riesgos del Trabajo (SRT) del Ministerio de Trabajo y Seguridad Social, con el objetivo de adaptar y validar el cuestionario. Dicho instrumento cuenta con una versión larga, una media y otra corta. Para nuestra validación se utilizó la versión larga de 95 ítems, los que se agrupan en 24 subdimensiones, que a su vez se ordenan en 7 dimensiones. En este artículo se presentan los resultados del proceso de adaptación cultural y la validación del cuestionario COPSOQ II para la población trabajadora de Argentina.

\section{Método}

El proceso de validación se diseñó de acuerdo con los procedimientos estandarizados para este tipo de estudios (Hernández Sampieri et al., 2010). En la primera etapa se realizó la adaptación cultural y la validez de contenido; y en la segunda, se llevo a cabo el estudio de validación del cuestionario, que incluyó la validación convergente de constructo y el estudio de fiabilidad. Durante todo el proceso se contó con el asesoramiento de los autores originales de la versión española.

\section{Instrumento y variables}

Entre los miembros del COPSOQ-ARG se revisó el marco teórico del cuestionario para evaluar la validez de contenido y se elaboró un manual con definiciones teóricas de las dimensiones y subdimensiones. La adaptación cultural se realizó en abril del 2015 a través de dieciocho entrevistas a asalariados según ocupación, edad, sexo y nivel educativo, a quienes se les preguntó acerca del sentido de sus respuestas. La muestra fue intencional, elegida con el criterio de muestreo de máxima variación (Zelaschi et al., 2016).

Las dificultades observadas en cuanto a la aplicación del cuestionario fueron en mayor medida respecto a la comprensión conceptual y a la claridad de los términos, 
notando que a un nivel de abstracción elevado, la confusión era mayor para quienes tenían un menor nivel de escolaridad. A partir de este hallazgo, se modificó la redacción de algunos ítems, el orden de algunas preguntas y se incluyó una guía de entrevista (Alonso et al., 1995).

Los cambios propuestos fueron discutidos y consolidados con los responsables del desarrollo de la versión española del cuestionario. Se concluyó que el cuestionario autoadministrado era muy difícil de completar entre los niveles de menor escolaridad.

Por lo tanto, para la etapa cuantitativa el cuestionario se aplicó en situación de entrevista, con encuestadores capacitados que tenían como guía un manual elaborado a tales fines. La versión utilizada fue la larga del cuestionario COPSOQIIISTAS21, compuesto por 7 dimensiones que contienen 24 subdimensiones y un total de 95 ítems. Cada uno posee cinco opciones de respuesta, en escala tipo Likert, con un rango de puntuación de 0 a 4 , siendo 4 la puntuación que indica mayor riesgo.

El cuestionario se administró incorporando dos apartados. En el primero se solicitaba al encuestado que respondiera sobre algunos aspectos socio-demográficos y de condiciones de trabajo que permitirían entender e identificar algunos aspectos de la muestra. Los datos solicitados incluían edad, sexo, máximo nivel educativo alcanzado, actividad económica principal de la organización a la cual pertenecía el entrevistado/a, número de trabajadores de dicho centro de trabajo, tipo de relación laboral que mantenía el trabajador con dicha organización y las horas semanales de trabajo. Mientras que el segundo apartado se focalizaba en cuestiones de salud. Para ello se utilizó la versión corta del cuestionario SF 36 (Hevia et al., 2009) para medir la calidad de vida de las personas, el estado de salud general, física y mental. La versión corta de este cuestionario cuenta con 12 ítems (SF12). La selección de este instrumento se basó, por un lado, por ser breve y, por otro, por haber sido validada en población argentina (Lévy Mangin y Varela Mallou, 2003).

\section{Diseño y muestra}

El diseño de la muestra fue aleatorio, estratificado y representativo de población asalariada, registrada en la seguridad social, que trabajaran en establecimientos con tres o más personas (se exceptuaron trabajadores/as domésticos/as). La estrategia de muestreo fue polietápica, representativa de cuatro municipios (Ciudad Autónoma de Buenos Aires, Avellaneda, Morón y Vicente López), tomando cuotas de sexo por partes iguales. Se seleccionaron, aleatoriamente, cinco conglomerados como puntos muestrales de cada municipio y se realizaron diez encuestas en cada punto. Las entrevistas domiciliarias se definieron azarosamente y las personas entrevistadas se seleccionaron incidentalmente si cumplían los criterios de inclusión antes mencionados. El trabajo de campo se hizo entre agosto y septiembre del 2015. La muestra fue de 200 trabajadores/as y su distribución por variables sociodemográficas se presenta en la Tabla 1. 


\begin{tabular}{|c|c|c|}
\hline Variables & $\mathbf{n}$ & $\%$ \\
\hline \multicolumn{3}{|l|}{ Sexo } \\
\hline Femenino & 97 & 48,5 \\
\hline Masculino & 103 & 51,5 \\
\hline \multicolumn{3}{|l|}{ Edad } \\
\hline $16-24$ & 21 & 10,5 \\
\hline $25-29$ & 25 & 12,5 \\
\hline $30-39$ & 54 & 27 \\
\hline $40-49$ & 43 & 21,5 \\
\hline $50-59$ & 42 & 21 \\
\hline $60-65$ & 15 & 7,5 \\
\hline \multicolumn{3}{|l|}{ Escolaridad } \\
\hline Primaria & 31 & 15,5 \\
\hline Secundaria & 96 & 48 \\
\hline Terciario & 35 & 17,5 \\
\hline Universitaria & 36 & 18 \\
\hline \multicolumn{3}{|l|}{ Actividad económica } \\
\hline Agricultura & 2 & 1 \\
\hline Industria & 23 & 11,5 \\
\hline Construcción & 5 & 2,5 \\
\hline Comercio y hostelería & 43 & 21,5 \\
\hline Transporte & 20 & 10 \\
\hline Servicios a la producción & 19 & 9,5 \\
\hline Servicios sociales & 88 & 44 \\
\hline \multicolumn{3}{|l|}{ Relación laboral } \\
\hline Estable & 170 & 85 \\
\hline Precarizado & 25 & 12,5 \\
\hline Autónomo & 5 & 2,5 \\
\hline
\end{tabular}

\section{Análisis de datos}

Se creó una nueva variable para cada ítem del cuestionario reemplazando las categorías de respuesta tipo Likert (4. Siempre, 3. Muchas veces, 2. Algunas veces, 1. Alguna vez, 0 . Nunca) por el puntaje que les correspondía según la valoración de la exposición de riesgo: la categoría "Siempre", representativa del mayor nivel de exposición, con un puntaje igual a 100, o "Nunca", simbólica del menor nivel de exposición, con un puntaje igual a 0 . En algunos ítems, este valor se invertía según el sentido de la pregunta.

Para el estudio de fiabilidad se utilizó la estrategia del test-retest. Una vez administrados los 200 casos, se retestearon 20 de ellos, para comprobar la consistencia del instrumento. Se calculó el coeficiente de correlación para cada ítem de las dimensiones por separado, entre los resultados del trabajo de campo y los resultados de la supervisión telefónica, sin observarse cambios (datos no mostrados).

La consistencia interna se evaluó aplicando el índice Alfa de Cronbach, calculado tanto para las dimensiones como para las subdimensiones. Partimos del supuesto que el COPSOQ es un cuestionario multidimensional, donde cada una de esas dimensiones es un constructo conceptual diferente, por lo tanto, la medición de los 
riesgos psicosociales derivados de estas se hace para cada dimensión por separado. En consecuencia, la evaluación de la estructura interna de los constructos se realizó a través del análisis factorial de cada dimensión. Este se efectuó con el método de componentes principales, con una rotación de tipo Varimax, incluyendo todas las dimensiones del cuestionario. Se excluyeron los factores que no superaron el criterio de la raíz latente y no se consideraron en la interpretación de contenidos las saturaciones inferiores a 0,30. La pertinencia de realizar un análisis factorial se evaluó con el índice de adecuación muestral de Kaiser-Meyer-Oklin (KMO) (Rosário et al., 2017).

El análisis de la validez convergente se realizó aplicando prueba de Chi-cuadrado (X2) entre los puntajes totales obtenidos en cada dimensión y subdimensión, con el puntaje del cuestionario SF-12 para salud general, mental y física. Para ello se dicotomizaron (expuesto/no expuesto) todas las dimensiones y subdimensiones del COPSOQ analizadas, de acuerdo al valor de la mediana. Del mismo modo se procedió dicotomizando (buena/mala) las variables de salud. El procesamiento de los datos se realizó con Statistical Packageforthe Social Sciences (SPSS) versión 20.

\section{Resultados}

En la Tabla 2 se muestran los resultados del análisis factorial para las dimensiones y subdimensiones, no se muestran las saturaciones individuales de los ítems. También se presentan los resultados del índice Alfa de Cronbach para valorar la consistencia interna.

La dimensión "Exigencias en el trabajo" presentó cinco factores con un $\mathrm{KMO}=$ 0,82 y una varianza acumulada de $60,1 \%$. El análisis factorial reproduce la estructura de subdimensiones de la propuesta original. Solo tres ítems mostraron saturaciones en dos componentes diferentes a la estructura conceptual, pero con saturaciones cercanas al 0,30 en los componentes ajenos. Tanto la dimensión como las subdimensiones presentaron $\alpha$ aceptables, o muy buenos.

La solución factorial que se extrajo en este estudio para la dimensión "Organización del trabajo" fue de siete factores que explican el $65 \%$ de la varianza acumulada, con un KMO de 0,80. Esta solución implica un factor más que la estructura de subdimensiones conceptuales propuesta. El problema se presenta en la subdimensión "Influencia" con saturaciones de sus ítems en dos factores diferentes, en lugar de un solo esperado: los últimos tres ítems se reagruparon de manera diferente al resto configurando una potencial subdimensión. Sin embargo, al analizar la consistencia interna de la subdimensión "Influencia" se encontró que era alta $(\alpha=0,827)$, incluso la más alta de toda la dimensión, razón por la cual se decidió conservar la dimensión completa con sus ítems originales.

"Relación interpersonaly liderazgo" es la dimensión que presentó la mayor disparidad respecto de la estructura original de ocho subdimensiones. Se obtuvieron seis factores con un KMO de 0,83 y una varianza del $61,3 \%$. "Calidad de liderazgo" se muestra como una dimensión desdibujada ya que sus ítems se confunden con el factor que 


\begin{tabular}{|c|c|c|c|c|c|c|}
\hline Factor & $\begin{array}{l}\text { \% Varianza } \\
\text { explicada }\end{array}$ & $\begin{array}{l}\text { \% Varianza } \\
\text { acumulada }\end{array}$ & $\begin{array}{c}\text { Dimensión y } \\
\text { subdimensiones }\end{array}$ & KMO & $\begin{array}{c}\text { Items } \\
\text { no }^{\circ}\end{array}$ & $\begin{array}{c}a \\
\text { Cronbach }\end{array}$ \\
\hline & & & Exigencias en el trabajo & 0,82 & 22 & 0,860 \\
\hline 1 & 27,88 & 27,88 & Cognitivas & & 6 & 0,821 \\
\hline 2 & 9,97 & 37,85 & Emocionales & & 5 & 0,810 \\
\hline 3 & 9,32 & 47,18 & Ritmo & & 3 & 0,811 \\
\hline 4 & 7,36 & 54,54 & Cuantitativas & & 4 & 0,735 \\
\hline \multirow[t]{2}{*}{5} & 5,97 & 60,51 & Esconder emociones & & 4 & 0,689 \\
\hline & & & Doble presencia & 0,66 & 4 & 0,746 \\
\hline \multirow[t]{2}{*}{1} & 56,92 & 56,92 & & & & \\
\hline & & & Organización del trabajo & 0,79 & 24 & 0,837 \\
\hline 1 & 22,95 & 22,95 & Posibilidad de desarrollo & & 4 & 0,805 \\
\hline 2 & 12,17 & 35,12 & Influencia & & 5 & 0,827 \\
\hline 3 & 7,83 & 42,95 & Compromiso & & 4 & 0,668 \\
\hline 4 & 7,70 & 50,65 & Control sobre los tiempos & & 3 & 0,745 \\
\hline 5 & 5,34 & 55,99 & Influencia & & 3 & \\
\hline 6 & 4,78 & 60,78 & Sentido del trabajo & & 3 & 0,674 \\
\hline \multirow[t]{2}{*}{7} & 4,22 & 65,00 & Variedad & & 2 & 0,631 \\
\hline & & & $\begin{array}{l}\text { Relación interpersonal y } \\
\text { liderazgo }\end{array}$ & 0,84 & 28 & 0,881 \\
\hline \multirow{2}{*}{1} & \multirow{2}{*}{26,12} & \multirow{2}{*}{26,12} & Calidad de liderazgo & & 5 & 0,865 \\
\hline & & & Apoyo superiores & & 3 & 0,594 \\
\hline \multirow{2}{*}{2} & \multirow{2}{*}{12,44} & \multirow{2}{*}{38,56} & Apoyo compañeros & & 3 & 0,732 \\
\hline & & & Sentimiento de grupo & & 3 & 0,844 \\
\hline 3 & 7,85 & 46,41 & Reconocimiento & & 4 & 0,766 \\
\hline 4 & 5,87 & 52,28 & Previsibilidad & & 2 & 0,636 \\
\hline 5 & 5,23 & 57,51 & Conflicto de rol & & 4 & \\
\hline \multirow[t]{2}{*}{6} & 3,80 & 61,31 & Claridad de rol & & 4 & 0,582 \\
\hline & & & Inestabilidad & 0,82 & 6 & 0,837 \\
\hline \multirow{3}{*}{1} & \multirow{3}{*}{55,27} & \multirow{3}{*}{55,27} & Externa & & 2 & 0,759 \\
\hline & & & Interna & & 4 & 0,817 \\
\hline & & & Confianza & 0,68 & 7 & 0,678 \\
\hline 1 & 35,71 & 35,71 & Vertical & & 4 & 0,631 \\
\hline \multirow[t]{2}{*}{2} & 19,40 & 55,11 & Horizontal & & 3 & 0,548 \\
\hline & & & Justicia & 0,80 & 4 & 0,839 \\
\hline 1 & 67,00 & 67,00 & & & 4 & \\
\hline
\end{tabular}

agrupa “Apoyo de superiores”. Pero además, posee saturaciones superiores al 0,30 en un segundo factor, pero esta vez compartido con la subdimensión "Previsibilidad". El a de "Apoyo de superiores" fue bajo al igual que el de "Previsibilidad". Por otra parte, la otra subdimensión que no presenta límites claros es "Sentimiento de grupo", ya que comparte componentes con "Apoyo de compañeros" y con "Reconocimiento", aunque siempre con saturaciones menores. Para estas subdimensiones los índices a fueron aceptables en todas.

La dimensión "Confianza" también presentó disparidades respecto a la propuesta conceptual original. Si bien se identificaron dos componentes, con un KMO de 0,68 y una varianza acumulada de 55,1\%, de manera coincidente con la estructura de las subdimensiones "Confianza vertical" y "Confianza horizontal", el problema surge 
con dos ítems cuyas saturaciones no corresponden con lo esperado. En el mismo sentido, los índices $a$ de Cronbach presentan valores bajos, tanto para la dimensión como para sus subdimensiones, especialmente, en la "Confianza horizontal".

"Inestabilidad" originalmente presentaba dos subdimensiones, "Externa" e "Interna", sin embargo en este estudio presentó un solo factor con $\mathrm{KMO}=0,82$ y el 55,3\% de la varianza. No obstante, tomadas por separado ambas dimensiones presentaron índices $\alpha$ adecuados.

Por último, las dimensiones que se proponen a nivel conceptual como unidimensionales, efectivamente, presentaron cargas factoriales en un solo factor: "Doble presencia", con un $\mathrm{KMO}=0,66$ y una varianza explicada del $57 \%$; y "Justicia", con el KMO de 0,80 y una varianza del $67 \%$. En ambos casos los índices a también fueron correctos.

Las características de la escala para cada dimensión y subdimiensión se presentan en la Tabla 3, donde se puede observar que todos los ítems presentan un poder de discriminación aceptable.

Finalmente, la validez convergente se expuso ante las hipótesis de vinculación de la exposición a riesgos psicosociales y su relación con mediciones de salud (Tabla 4). Todas las dimensiones del cuestionario se relacionan de manera esperada en cuanto a la percepción que tiene los sujetos de su "mala salud general", siendo estas relaciones estadísticamente significativas en cuatro de ellas: "Organización del trabajo", "Relación interpersonal y liderazgo", "Confianza" y "Justicia”. La hipótesis de validación se mantiene para la mayoría de las dimensiones del COPSOQ-ARG, si consideramos la mala salud mental, a excepción de "Exigencias del trabajo" e "Inestabilidad". Finalmente, si tomamos la percepción de mala salud física, solo los peores puntajes de "Confianza" y "Justicia" mantienen las relaciones esperadas.

\section{Discusión}

El proceso de adaptación cultural y de validación del COPSOQ-ARG respondió a los procedimientos habituales y completó todas las etapas necesarias para tal fin. La revisión de la estructura conceptual del cuestionario entre el grupo de expertos y la supervisión de los autores de la versión en castellano, permitieron reforzar los consensos internacionales en torno a la validez de contenido. Si bien, durante la adaptación cultural no se presentaron grandes dificultades de comprensión, el principal resultado de la misma fue que entre las personas con menores niveles de escolaridad la dinámica de lectura y comprensión se dificultaba; por lo tanto, se decidió realizar la validación del cuestionario en forma de entrevista con encuestadores, en lugar del cuestionario autoadministrado. Esto presenta desafíos en un futuro para la aplicación en Argentina del método original.

El análisis de la estructura del instrumento pudo reproducir la estructura de las siete dimensiones conceptuales a través del análisis factorial, que presenta una ventaja para el uso de la versión corta del COPSOQ II. No obstante, en el nivel de 
Tabla 3. Media, desvío estándar, valores mínimos y máximos de las dimensiones y subdimensiones COPSOQ-ARG

\begin{tabular}{|c|c|c|c|c|}
\hline $\begin{array}{c}\text { Dimensión y } \\
\text { subdimensiones }\end{array}$ & Media & $\begin{array}{c}\text { Desvío } \\
\text { estándar }\end{array}$ & $\begin{array}{c}\text { \% valores } \\
\text { piso }\end{array}$ & $\begin{array}{c}\% \text { valores } \\
\text { techo }\end{array}$ \\
\hline \multicolumn{5}{|l|}{ Exigencias en el trabajo } \\
\hline Cognitivas & 59,7 & 25,0 & 10,5 & 35,5 \\
\hline Emocionales & 43,2 & 26,5 & 40,5 & 10,5 \\
\hline Ritmo & 51,8 & 28,7 & 12,0 & 26,5 \\
\hline Cuantitativas & 30,9 & 22,6 & 48,0 & 2,0 \\
\hline Esconder emociones & 43,0 & 27,7 & 55,0 & 5,5 \\
\hline \multicolumn{5}{|l|}{ Doble presencia } \\
\hline & 39,3 & 24,4 & 33,5 & 7,0 \\
\hline \multicolumn{5}{|l|}{ Organización del trabajo } \\
\hline Posibilidad de desarrollo & 27,5 & 25,5 & 7,5 & 52,5 \\
\hline Influencia & 51,4 & 23,8 & 47,0 & 13,0 \\
\hline Compromiso & 35,1 & 24,7 & 7,0 & 39,5 \\
\hline Control sobre los tiempos & 42,5 & 29,6 & 15,0 & 31,5 \\
\hline Sentido del trabajo & 14,2 & 16,5 & 1,5 & 67,0 \\
\hline Variedad & 57,2 & 27,8 & 7,0 & 34,5 \\
\hline \multicolumn{5}{|c|}{ Relación interpersonal y liderazgo } \\
\hline Calidad de liderazgo & 38,9 & 27,3 & 7,5 & 33,0 \\
\hline Apoyo superiores & 36,4 & 27,2 & 5,5 & 41,0 \\
\hline Apoyo compañeros & 29,0 & 22,9 & 3,5 & 41,0 \\
\hline Sentimiento de grupo & 18,6 & 21,9 & 1,5 & 53,0 \\
\hline Reconocimiento & 34,4 & 24,6 & 5,5 & 44,0 \\
\hline Previsibilidad & 43,3 & 29,0 & 9,0 & 31,5 \\
\hline Conflicto de rol & 38,7 & 25,2 & 53,5 & 5,5 \\
\hline Claridad de rol & 19,4 & 16,4 & 0,5 & 68 \\
\hline \multicolumn{5}{|l|}{ Inestabilidad } \\
\hline Externa & 28,8 & 30,9 & 59,0 & 7,0 \\
\hline Interna & 25,5 & 28,9 & 66,0 & 11,0 \\
\hline \multicolumn{5}{|l|}{ Confianza } \\
\hline Vertical & 35,7 & 21,3 & 4,5 & 43,0 \\
\hline Horizontal & 35,0 & 22,8 & 2,0 & 46,5 \\
\hline \multicolumn{5}{|l|}{ Justicia } \\
\hline & 39,5 & 25,5 & 3,0 & 21,0 \\
\hline
\end{tabular}

Tabla 4. Relación de las dimensiones del COPSOQ-ARG con salud general, salud mental y salud física

\begin{tabular}{llrrrrr}
\hline \multirow{2}{*}{ Dimensión } & \multicolumn{2}{c}{ Salud general } & \multicolumn{2}{c}{ Salud mental } & \multicolumn{2}{c}{ Salud física } \\
\cline { 2 - 7 } & \multicolumn{1}{c}{$\mathbf{X}^{\mathbf{2}}$} & $\mathbf{p}$ & $\mathbf{X}^{\mathbf{2}}$ & $\mathbf{p}$ & $\mathbf{X}^{\mathbf{2}}$ & $\mathbf{p}$ \\
\hline Exigencias en el trabajo & $1,457^{*}$ & 0,227 & $0,631^{*}$ & 0,427 & $0,455^{\star}$ & 0,500 \\
Doble presencia & $1,245^{*}$ & 0,265 & $2,903^{\star}$ & 0,880 & $0,099^{*}$ & 0,753 \\
Organización del trabajo & 4,895 & 0,027 & 5,259 & 0,022 & $2,465^{*}$ & 0,116 \\
Relación interpersonal y liderazgo & 8,158 & 0,004 & 10,902 & 0,001 & $2,465^{*}$ & 0,116 \\
Inestabilidad & $1,245^{*}$ & 0,264 & $0,728^{*}$ & 0,394 & $2,080^{*}$ & 0,149 \\
Confianza & 12,36 & 0 & $3,546^{*}$ & 0,600 & 6,016 & 0,140 \\
Justicia & 9,165 & 0,002 & 5,837 & 0,016 & 8,130 & 0,004 \\
\hline
\end{tabular}

Nota: Chi-cuadrado significativo a nivel de $\mathrm{p}<0,005$ y $\mathrm{R}>3,841 .{ }^{*}$ Estadísticamente no significativo. 
subdimensiones surgieron dificultades en la reproducción de la estructura general, lo que resulta coincidente con resultados de otros estudios de validación. El uso de las siguientes subdimensiones, deberá ser analizado en cada caso en particular hasta que se puedan realizar estudios en muestras más potentes.

En el caso de "Organización del trabajo" es la subdimensión "Influencia” la que se desdobla en dos factores en lugar de uno: tres ítems remanentes se separan con el $5 \%$ de la varianza explicada. Esta dimensión también ha presentado dificultades en otros estudios.

Similar es el resultado en "Relaciones interpersonales y liderazgo". Las saturaciones de la subdimensión "Liderazgo" se confunde con "Apoyo de Superiores" y con "Previsibilidad"; mientras que "Sentimiento de grupo" hace lo propio con "Apoyo de compañeros" y con "Reconocimiento". En este caso, como muestra la bibliografía, las subdimensiones parecen agrupar aquellos ítems que evalúan las tareas de supervisión, por un lado y los que refieren al grupo de trabajadores/as, por el otro. Incluso en el estudio de Alvarado et al. (2012), se propone una reagrupación de los ítems en dos subdimensiones nuevas: calidad de relaciones con superiores y calidad de relaciones con los compañeros. En futuros estudios habrá que explorar la estructura más adecuada para la aplicación del cuestionario.

En la dimensión "Confianza" el análisis factorial no reprodujo los factores de "Confianza vertical” y "Confianza horizontal”, sino que se reagruparon en lo que podríamos denominar como "confianza" o "desconfianza", sin importar de quién se trate.

Por último, en "Inestabilidad" todos los ítems se agruparon en un solo factor. Esto indicaría que no sería relevante la diferenciación externa o interna de la inestabilidad, sino una percepción de inestabilidad general. Debemos tener en cuenta que más del $85 \%$ de la muestra estaba compuesta por trabajadores efectivos, lo que reduce la percepción de la "Inestabilidad externa".

En cuanto a la consistencia interna fue óptima y mejoró cuando se eliminaron algunos ítems del cuestionario original. Seis dimensiones obtuvieron un alfa de Cronbach mayor que el nivel convencional de 0,700 y cinco mayor a 0,800. No obstante, aquellas subdimensiones que presentaron disonancias en el análisis factorial, también mostraron valores a menores al convencional "Apoyo de superiores", "Previsibilidad”, “Claridad de rol”, "Confianza vertical y horizontal”).

El análisis convergente mostró las relaciones entre las dimensiones del cuestionario y los índices de salud en el SF 12. Sin embargo, la cantidad de dimensiones con relaciones estadísticamente significativas, disminuyen según la variable de salud que se considere: con salud general, cuatro dimensiones, con salud mental, tres y salud física, solo dos.

Es necesario recordar que el COPSOQ II es un instrumento que mide factores psicosociales del trabajo a partir del cual se inicia un proceso participativo de valoración del riesgo y una planificación preventiva. Es decir, el método requiere condiciones laborales previas a su aplicación, que favorezcan el diálogo, la negociación y el consenso. Esas precondiciones suelen ser infrecuentes en un mercado de trabajo 
precarizado, con fuerte disciplinamiento de la fuerza de trabajo y reducidos derechos de participación por la salud de los/as trabajadores/as. Es por ello que es necesario un trabajo previo de presentación del instrumento. Además, existen condiciones técnicas para su aplicación que implican una incorporación legal plena de los riesgos psicosociales en las evaluaciones de riesgos y una mayor interdisciplinariedad en la gestión de la salud laboral. Ambos requisitos, sociales y técnicos, son necesarios para evitar un uso tecnocrático del cuestionario.

No obstante, consideramos que la versión del COPSOQ-ARG permite evaluar la presencia de riesgos psicosociales en el trabajo, siendo un instrumento equivalente a las versiones internacionales que le anteceden. La validación de este instrumento debe continuar considerando su desempeño en realidades laborales diversas. Es de esperar que el marco legislativo propicie y resguarde la salud de los trabajadores en relación con los riesgos psicosociales del trabajo. Mientras tanto, los/as trabajadores/as, sus representantes y empleadores, así como asesores/ as técnicos/as y profesionales, disponen de una metodología para comenzar a mejorar el bienestar y la salud en el trabajo.

\section{Limitaciones y futuras líneas de investigación}

Los resultados del proceso de validación del COPSOQII-ISTAS 21 son óptimos teniendo en cuenta las limitaciones de la metodología utilizada. Se optó por generar una muestra de población trabajadora por conglomerado geográfico sin estratificar por variables ocupacionales y con escasa potencia estadística. Este último aspecto es una clara limitación del estudio, como así también la distribución de la muestra entre tantos ítems, lo que debilita los resultados obtenidos.

Por lo antedicho, la validación del cuestionario será un proceso permanente de mejora en la evaluación de su estabilidad, aspecto importante para su interpretación. La posibilidad de obtener una muestra mayor con representación de distintos sectores laborales y ocupaciones permitirá tener mejores parámetros de aplicación. Asimismo, el beneficio que puede tener una muestra representativa de población trabajadora general hace que, a su vez, resulte más heterogénea en lo ocupacional. Esa diversidad laboral dificulta la interpretación de los resultados en esta instancia de validación. A partir de la utilización del cuestionario en el futuro se podrá continuar revisando algunos resultados de este estudio.

Entre los aspectos a tener en cuenta en la aplicación del instrumento, deberá considerarse la simplificación de algunas preguntas, contemplando la dificultad en la comprensión de las mismas en función a los niveles educativos de la población encuestada.

En cuanto a la estructura del cuestionario, se deberá continuar observando el comportamiento de algunas dimensiones y subdimensiones que no responden claramente a lo esperado. Una de ellas es "Confianza" que no responde a aspectos horizontales (pares) o verticales (jefes), sino a la percepción de confianza o desconfianza generada en el lugar de trabajo. En este caso no se encuentran 
antecedentes de la bibliografía por lo que se deberán hacer estudios en profundidad para comprender a qué responde.

Algunas de estas limitaciones que presenta el estudio, conllevan a continuar observando el comportamiento del instrumento, tanto en su estructura como en la identificación de aspectos diferenciales que podrían estar respondiendo a las particularidades de los sectores de actividad de nuestra población.

\section{Agradecimientos}

En Argentina, a partir de 2013 se conformó el grupo COPSOQ-ARG entre la Universidad Nacional de Avellaneda (UNDAV, UNDAVCYT 2012, PICTO 100) y la Superintendencia de Riesgos del Trabajo (SRT, Exp 90877-15), Ministerio de Trabajo y Seguridad Social, con el objetivo de validar la versión larga del instrumento. Agradecemos la colaboración de Johana Gerke, Alberto Marcos y Adela Contreras de la superintendencia, quienes han participado en la primera etapa de este trabajo.

\section{Referencias}

Alonso, J., Prieto, L. y Antó, J. M. (1995). La versión española del SF-36 Health Survey (Cuestionario de Salud SF-36): un instrumento para la medida de los resultados clínicos. Medicina Clínica, 104(20), 771-776

Alvarado, R., Pérez-Franco, J., Saavedra, N., Fuentealba, C., Alarcón, A., Marchetti, N., y Aranda, W. (2012). Validación de un cuestionario para evaluar riesgos psicosociales en el ambiente laboral en Chile. Revista médica de Chile, 140(9), 1154-1163. HTTPS://DX.DOI.ORG/10.4067/So034$\underline{98872012000900008}$

Amable, M. (2006). La precariedad laboral y su impacto en la salud. Un estudio en trabajadores asalariados en España. Tesis Doctoral. Pompeau Fabra. HTTP://HDL.HANDLE.NET/10803/7116

Belkic, K., Landsbergis, P.A., Schnall, P.L. y Baker, D. (2004). Is job strain a major source of cardiovascular risk? Scandinavian Journal of Work, Environment \& Health, 30(2), 85-128. HTTPS://DOI.ORG/10.5271/SIWEH.769

Benach, J., Amable, M., Muntaner, C. y Benavides, F.G. (2002). The consequences of flexible work for health: Are we looking at the right place? Journal of Epidemiology \& Community Health, 56, 405406.
Benavides, F. G., Ruiz-Frutos, C. y García, A. M. (2007). Trabajo y salud. En C. Ruiz-Frutos, A. M. García, J. Delclós y F. G. Benavides (Eds.), Salud laboral. Conceptos y técnicas para la prevención de riesgos laborales (pp. 33-41). Masson.

Benavides, M., López-Ruiz, M., Rojas, M., SilvaPeña, C., Herrera, M., Chavarria, J., Cornelio, C. y Rodríguez-Guzmán, J. (2018). Brechas de desigualdad en la salud de las personas que trabajan. Informe de salud ocupacional en América Latina y el Caribe. Serie Salud y Trabajo No 35. SALTRA / IRET-UNA.

Benzoni, P. E. (2018). A influência do estresse na condição de afastamento do trabalho por distúrbios osteomusculares. Gerais: Revista Interinstitucional de Psicologia, 11(2), 294-305. HTTPS://DX.DOI.ORG/10.36298/GERAIS2019110208

Cardoso, J., Araújo, T., Carvalho, F., Fernandes de Oliveira, N. y Farias Borges dos Reis, E. (2011). Aspectos psicossociais do trabalho e dor musculoesquelética em professores. Cadernos de Saúde Pública, 27(8), 1498-1506. HTTPS://DOI. ORG/10.1590/So102-311X2011000800005

CSDH (2008). Closing the gap in a generation: health equity through action on the social determinants of health. Final report: Executive summary. Organización Mundial de la Salud. HTTPS://APPS. WHO.INT/IRIS/HANDLE/10665/69832 
Ferrie, J. E. (2001). Is job insecurity harmful to health? Journal of the Royal Society of Medicine, 94(2), 71-76. HTTPS://DOI. ORG/10.1177/014107680109400206

Gimeno, D., Benavides, F.G., Amick III, B., Benach, J. y Martınez, J.M. (2004). Psychosocial factors and work related sickness absence among permanent and non-permanent employees. Journal of Epidemiology and Community Health, $58,870-876$.

Gómez-Ortiz, V. y Moreno, L. (2009). Factores psicosociales del trabajo (demanda-control y desbalance esfuerzo-recompensa), salud mental y tensión arterial: un estudio con maestros escolares en Bogotá, Colombia. Universitas Psychologica, 9(2), 393-407.

Hernández Sampieri, R., Fernández Collado, C., Baptista Lucio M. del P., (2010). Metodología de la investigación ( $5^{\mathrm{a}} \mathrm{ed}$. .). McGraw Hill.

Hevia, I., Gomez Saldaño, A. M., Cavalieri, L., Dawidowski, A., Figar, S., Beratarrechea, A. y Marchetti, M. (2009). Calidad de vida en adultos mayores de 65 años: adaptación del cuestionario SF12. Programa de Enfermedades Crónicas. Sección Investigación Cualitativa. Área de Epidemiología de la Gerencia Médica del Plan de Salud. Servicio de Clínica Médica. Hospital Italiano de Buenos Aires.

Kalimo, R., El-Batawi, M. y Cooper, C. (Comps.) (1988). Los factores psicosociales en el trabajo $y$ su relación con la salud. Organización Mundial de la Salud. HTTPS://APPS.WHO.INT/IRIS/ HANDLE/10665/37881

Karasek, R. (1979). Job demands, job decision latitude and mental strain: implications for job redesign. Administrative Science Quarterly, 24, 285-308. HTTPS://WWW.JSTOR.ORG/STABLE/2392498

Kivimäki, M., Leino-Arjas, P., Luukkonen, R., Riihimäki, H., Vahtera, J. y Kirjonen, J. (2002). Work stress and risk of cardiovascular mortality: Prospective cohort study of industrial employees. BMJ, 325, 857. HTTPS://DOI.ORG/10.1136/ BMJ.325.7369.857

Kristensen, T. (2010). A questionnaire is more than a questionnaire. Scandinavian Journal of Public Health, 38(3), 149-155.

Kristensen, T., Hannerz, H., Hogh, A. y Borg, V. (2005). The Copenhagen Psychosocial Questionnaire, a tool for assessment and improvement of the psychosocial work environment. Scandinavian Journal of Work, Environment \& Health, 31(6), 438-449. HTTPS:// DOI.ORG/10.5271/SJWEH.948
Landsbergis, P. y Theorell, T. (2000). Measurement of psychosocial workplace exposure variables. Occupational Medicine, 15(1), 7-68.

Lévy Mangin, J. y Varela Mallou, J. (2003). Análisis multivariable para las ciencias sociales. Pearson.

Lima, I., Parma, G., Cotrim, T. y Moro, A. (2019). Psychometric properties of a medium version of the Copenhagen Psychosocial Questionnaire (COPSOQ II) for southern Brazil. Work, 62(2), 175-184.

Moncada, S., Llorens, C., Andrés, R., Moreno, N., y Molinero, E. (2014). Manual del método CoPsoQ-istas21 (versión 2) para la evaluación y la prevención de los riesgos psicosociales en empresas con 25 o más trabajadores y trabajadoras. Versión media. ISTAS CCOO.

Moncada, S., Llorens, C., Navarro, A. y Kriestensen, T. (2005). ISTAS21: Versión en lengua castellana del cuestionario psicosocial de Copenhague (COPSOQ). Archivos de Prevención de Riesgos Laborales, 8(1), 18-29.

Moncada, S., Llorens Serrano, C., Font Corominas, A., Galtés Camps, A. y Navarro Giné, A. (2008). Exposición a riesgos psicosociales entre la población asalariada en España (2004-05): valores de referencia de las 21 dimensiones del cuestionario COPSOQ ISTAS21. Revista Española de Salud Pública, 82(6), 667-675.

Nolle, I. y Kranich, J. (2020). List of publications with COPSOQ published in peer-reviewed indexed journals. COPSOQ International Network.

Paoli, P. (1992). First European survey on the work environment 1991-1992. Fundación Europea para la Mejora de las Condiciones de Vida y de Trabajo.

Ramada-Rodilla, J. M., Serra-Pujadas, C. y DelclósClanchet, G.L. (2013). Adaptación cultural y validación de cuestionarios de salud: revisión y recomendaciones metodológicas. Salud Publica México, 55(1), 57-66.

Rosário, S., Azevedo, L. F., Fonseca, J. A., Nienhaus, A., Nübling, M. y Torres da Costa, J. (2017). The Portuguese long version of the Copenhagen Psychosocial Questionnaire II (COPSOQ II) - A validation study. Journal of Occupational Medicine and Toxicology, 12, 24. HTTP://DX.DOI. ORG/10.1186/s12995-017-0170-9

Siegrist, J., Peter, R., Junge, A., Cremer, P. y Seidel, D. (1990). Low status control, high effort at work and ischemic heart disease: prospective evidence from blue-collar men. Social Science \& Medicine, 31(10), 1127-1134. HTTPs://DOI. ORG/10.1016/0277-9536(90)90234-I 
Superintendencia de Riesgos del Trabajo (2009). 1era encuesta nacional a trabajadores sobre empleo, trabajo y condiciones y medio ambiente laboral. Ministerio de Trabajo, Empleo y Seguridad Social. HTTPS://WWW.ARGENTINA.GOB.AR/ SITES/DEFAULT/FILES/PRIMERA_ENCUESTA_ NACIONAL TRABAJADORES.PDF

Virtanen, M., Kivimäki, M., Joensuu, M., Virtanen, P., Elovainio, M. y Vahtera, J. (2005). Temporary employment and health: A review. International Journal of Epidemiology, 34(3), 610-622. HTTPs:// DOI.ORG/10.1093/IJE/DYIO24

Zárate Castillo, B. G. y Gómez Gómez, M. (2012). Validación del cuestionario COPSOQ-ISTAS21 en personal de salud del HGZMF-21 del IMSS en León, Guanajuato, México. IV Simposio internacional sobre Salud Ocupacional: Convención Internacional de Salud Pública. Cuba Salud.
Zelaschi, M. C., Amable, M., Gerke, J., Cornelio, C., Alberto, M., Contreras, A., Reif, L. y Gonzalez Francese, R. (3-5 de agosto de 2016). Estudios de adaptación cultural para la Argentina del COPSOQ - ISTAS21. VIII Congreso ALAST. Universidad de Buenos Aires, Argentina.

Zelaschi, M. C. (2010). Inestabilidad laboral, riesgos psicosociales y padecimientos en el trabajo. Caso de los trabajadores operarios metalúrgicos de Villa Constitución. Tesis doctoral inédita. Facultad de Ciencias Sociales, Universidad de Buenos Aires. 